\title{
Lock-In Amplifier Technology in Laser Gyroscope North Finder of Constant Rate Biasing
}

\author{
Bin Wang, ${ }^{1}$ Wei Zhang, ${ }^{2}$ Zhanqing Wang, ${ }^{3}$ and Peihao Zhu ${ }^{1}$ \\ ${ }^{1}$ School of Mechanical Engineering, Tianjin University, Tianjin 300072, China \\ ${ }^{2}$ School of Underwater Acoustic Engineering, Harbin Engineering University, Harbin 150001, China \\ ${ }^{3}$ School of Automation, Beijing Institute of Technology, Beijing 100081, China \\ Correspondence should be addressed to Bin Wang; wang_bin@tju.edu.cn
}

Received 21 January 2013; Accepted 11 March 2013

Academic Editor: Cristian Toma

Copyright (C) 2013 Bin Wang et al. This is an open access article distributed under the Creative Commons Attribution License, which permits unrestricted use, distribution, and reproduction in any medium, provided the original work is properly cited.

\begin{abstract}
This paper presents a new type of strapdown north finder. A simplified configuration is proposed, including three parts: a pendulous force-feedback accelerometer, a ring laser gyroscope, and a single-axis rotating platform. The dynamic northfinding scheme of continuous rotation is adopted to eliminate the lock-in region of the laser gyroscope and to modulate the weak attitude signals; meanwhile the complex leveling process is avoided in this proposed configuration. To suppress the drift noises of the accelerometer and gyroscope, two digital lock-in amplifiers are used to extract the weak attitude signals. Simulation results show that the north finder can determine the heading angle in $72 \mathrm{~s}$, when the rotating rate is $10^{\circ} / \mathrm{s}$ with the maximum heading error less than $1.0^{\prime}$; meanwhile the horizontal attitude angles as well as the constant drift noises of the inertial components could also be obtained.
\end{abstract}

\section{Introduction}

The equipment mounted in the body of the land-based vehicle would allow the directional reference with respect to geographical true north. This equipment is often referred to as north finder, which plays an important role in both civilian and military fields [1-6]. According to the principles of north finding, there are two kinds of north finders: non-gyro-north finder and gyroscope north finder.

In the non-gyro-north finder, magnetic compass and astronomical navigator are the two major categories. Magnetic compass enables the vehicle's attitude to be determined with respect to the Earth's magnetic field; the astronomical navigator fixed in the body of a vehicle could provide measurements of the remote star's bearing and elevation with respect to the body frame relying on the optical sensors. But these two instruments are highly sensitive to the external environment, such as magnetic field, weather, and sometimes cannot be used any more.

In view of the limitations of both the aforementioned techniques, the gyroscope north finder has the characteristic of autonomy, which is not impacted by external environment; therefore more attentions have been paid to these greatest inventions. With the development of the inertial components, the gyroscope north finder is mainly divided into two categories: one is pendulous north finder as the representative of mechanical gyroscope, which is based on the principle of the gyrocompass with high accuracy but long alignment time. The basic principle of this equipment is the indication of true north by establishing the equilibrium between the effect of its pendulosity and the angular momentum of the rotation base carrying the mechanical gyroscope; the other is strapdown north finder using the optical gyroscope as a sensor [1,2]. Usually, the strapdown north finder keeps the sensitive axis of the optical gyroscope in the horizontal plane accomplished by using the outputs of two orthogonal accelerations to drive the motor, respectively. Two measurements of the horizontal components of the Earth's rate are sensed by optical gyroscope in two separations $90^{\circ}$ apart. So the heading angle can be calculated from the ratio of these two measurements. The strapdown north finder locates the orientation with a little 
lower accuracy to the pendulous north finder but greatly decreases the time.

Through analyzing the dynamic output characteristics of inertial measurement unit in the presence of continuous rotation at a constant rate, this paper designs a new type of strapdown north finder with a simplified configuration and also can avoid the complex leveling process of rotating platform. Digital lock-in amplifier technology is adopted to extract the weak attitude signals submerged in the drift noise of inertial components effectively [7-10]. Three attitude angles are obtained in a short time with certain accuracy, as well as the estimations of the constant drifts of inertial components.

\section{The Dynamic Output Characteristics of the Inertial Components at Constant Rate Rotation}

2.1. The Measurement Principle of Laser Gyroscope of Constant Rate Biasing. The ring laser gyroscope (RLG) based on the Sagnac effect is an angular rate sensor, being an important inertial component currently. The RLG relies upon the detection of the frequency difference between the two counter-propagating beams of light in the optical resonant cavity, which is also proportional to the rotational motion about an axis perpendicular to the plane containing the light path in inertial space. It appears that the application of RLG to the sensing of angular rate can offer lots of advantages over the mechanical gyroscope, such as rapid response, wide dynamic detecting range, good linearity, little dynamic error, high precision, and high reliability and non- $g$-dependent bias term. Therefore, RLG gets more and more attentions and now is considered as the ideal inertial component to replace the well-established mechanical gyroscope.

At very low angular rate, due to a variety of nonuniformity factors in the optical resonant cavity of RLG, the two counter propagating beams almost have the same frequency or "lock together," so there is no output signal, which is usually called the lock-in phenomenon [11, 12], as shown in Figure 1. This phenomenon of frequency synchronization introduces a dead zone of the RLG measurement signal, so how to overcome the lock-in region becomes one of the key questions gradually. Although mechanical dither technology is widely used in RLG, it cannot eliminate the lock-in region completely, the components of which still cause measurement error in the mechanical dither.

Fortunately, the lock-in region could be eliminated completely by employing a so-called constant rate biasing technology which is superior to mechanical dither in principle [13]. RLG is installed on a platform with an appropriate rotating rate, which can make a projection rate on the sensitive axis of RLG, so the output signal can keep away from measurement error induced by the lock-in region. Theoretically, the faster the platform rotates, the smaller the laser measurement error is, but a faster rate will reduce the stability of the rotating platform and gyroscope sampling points in a whole rotation cycle, which will affect the calculation accuracy of the attitude solution. Usually, to keep

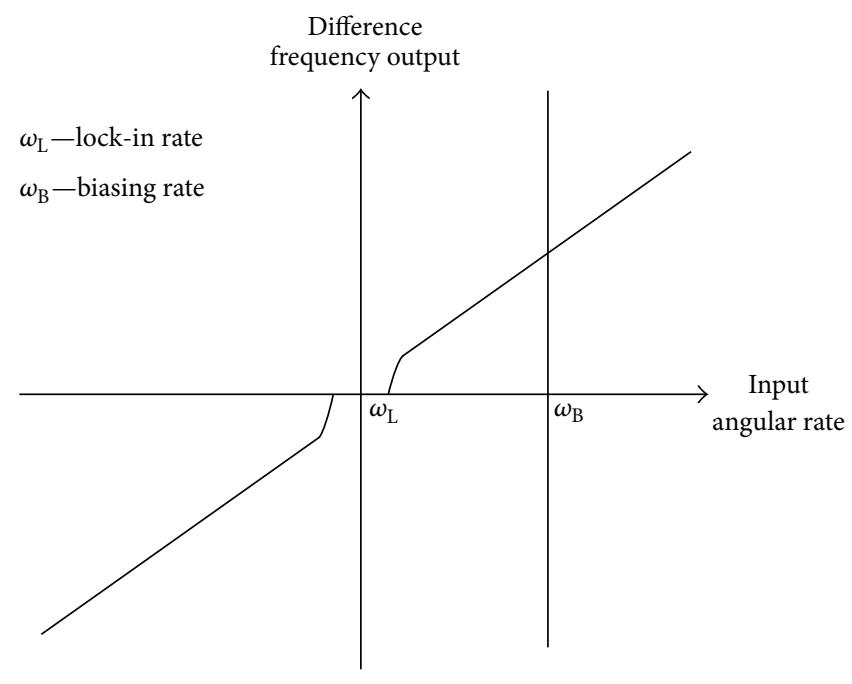

FIGURE 1: The RLG input/output characteristic.

the measurement of RLG mounted on the rotating platform away from the lock-in region, the biasing rate $\left(\omega_{B}\right)$ provided by the platform should be ten to twenty times that of the lockin rate $\left(\omega_{L}\right)$.

2.2. The Dynamic Output Characteristics of Inertial Measurement Unit at Constant Rate Rotation. The Cartesian coordinate frames used in this paper are defined as follows $[14,15]$.

The inertial frame ( $i$-frame) sets its origin $(o)$ at the center of the Earth and axes $\left(o x_{i}, o y_{i}\right.$, and $\left.o z_{i}\right)$ which are invariant in direction with respect to the fixed planets in space. In $i$-frame, axes $o x_{i}$ and $o y_{i}$ are parallel to the equator plane, with axis $o x_{i}$ coinciding with the spring equinox and axis $o z_{i}$ coinciding with the Earth's polar axis, respectively.

The Earth frame (e-frame) sets origin $(o)$ at the center of the Earth and axes $\left(o x_{e}, o y_{e}\right.$, and $\left.o z_{e}\right)$ which are in respect to the Earth. In $e$-frame, axes $o x_{e}$ and $o y_{e}$ are parallel to the equator plane, with axis $o x_{e}$ along the $0^{\circ}$ longitude, axis $o y_{e}$ along the $90^{\circ}$ longitude, and axis $o z_{e}$ coincident with the Earth's polar axis, respectively. The $e$-frame rotates relative to the $i$-frame at a rate $\omega_{i e}$ about the axis $o z_{e}\left(o z_{i}\right)$.

The navigation frame ( $n$-frame), the reference frame for the attitude solution, is a local geographic one. Its origin $(o)$ is set at the location of the navigation system. And axes $\left(o x_{n}, o y_{n}\right.$, and $\left.o z_{n}\right)$ lie along the directions of the local east, the local north, and the vertical upturn, respectively.

The body frame ( $b$-frame) is attached to the base of the vehicle with its origin $(o)$ located at the center of vehicle. Axes $\left(o x_{b}, o y_{b}\right.$, and $\left.o z_{b}\right)$ lie along the directions of the pitch (right), roll (front), and yaw (upturn) of the vehicle, respectively.

The gyroscopes frame ( $g$-frame) is defined by the axes $\left(o x_{g}, o y_{g}\right.$, and $\left.o z_{g}\right)$ which are parallel to the sensitive axes of the three gyroscopes, respectively.

The accelerometers frame ( $a$-frame) is defined by the axes $\left(o x_{a}, o y_{a}\right.$, and $\left.o z_{a}\right)$ which are parallel to the sensitive axes of the three accelerometers, respectively. 


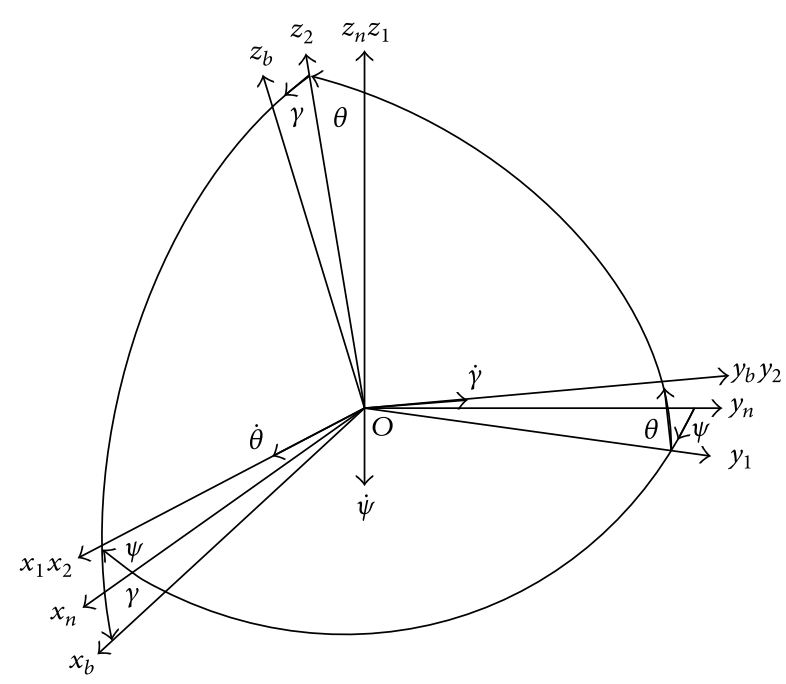

FIGURE 2: The transformation from $n$-frame to $b$-frame.

Moreover, the coordinate transformation is defined as $V^{2}=C_{1}^{2} V^{1} . V^{1}, V^{2}$ represent vectors expressed in the 1frame, 2-frame, respectively, and $C_{1}^{2}$ is the direction cosine matrix transferring $V^{1}$ into $V^{2}$. The $\omega_{12}^{3}$ represents the angular rate of the 2 -frame with respect to the 1-frame expressed in the 3 -frame. The positive rotations about each axis are taken to be in a clockwise direction looking along the axis from the origin.

The vehicle's attitude matrix $C_{n}^{b}$ is the transform matrix from $n$-frame to $b$-frame by three successive rotations about heading axis, pitch axis, and roll axis in turn, as shown in Figure 2:

$$
\begin{gathered}
o-x_{n} y_{n} z_{n} \stackrel{\operatorname{Rot}\left(\Psi,{ }^{-} z_{n}\right)}{\longrightarrow} o-x_{1} y_{1} z_{1} \stackrel{\operatorname{Rot}\left(\theta, x_{1}\right)}{\longrightarrow} o-x_{2} y_{2} z_{2} \\
\stackrel{\operatorname{Rot}\left(\gamma, y_{2}\right)}{\longrightarrow} o-x_{b} y_{b} z_{b} .
\end{gathered}
$$

Rotate through heading angle $\psi$ about reference $-z_{n}$ axis, $\psi \in\left[0^{\circ}, 360^{\circ}\right)$.

Rotate through pitch angle $\theta$ about new $x_{1}$-axis, $\theta \in$ $\left[-90^{\circ}, 90^{\circ}\right]$.

Rotate through roll angle $\gamma$ about new $y_{2}$-axis, $\gamma \in$ $\left[-90^{\circ}, 90^{\circ}\right]$.

where $\psi, \theta$, and $\gamma$ are referred to as the attitude angles of the vehicle. Usually, $\theta$ and $\gamma$ represent the horizontal attitude angles. follows:

Thus the direction cosine matrix $C_{n}^{b}$ could be expressed as

$$
C_{n}^{b}=C_{2}^{b} C_{1}^{2} C_{n}^{1}
$$

where

$$
\begin{gathered}
C_{2}^{b=}\left[\begin{array}{ccc}
\cos \gamma & 0 & -\sin \gamma \\
0 & 1 & 0 \\
\sin \gamma & 0 & \cos \gamma
\end{array}\right], \quad C_{1}^{2}=\left[\begin{array}{ccc}
1 & 0 & 0 \\
0 & \cos \theta & \sin \theta \\
0 & -\sin \theta & \cos \theta
\end{array}\right], \\
C_{n}^{1}=\left[\begin{array}{ccc}
\cos \psi & -\sin \psi & 0 \\
\sin \psi & \cos \psi & 0 \\
0 & 0 & 1
\end{array}\right] .
\end{gathered}
$$

Substituting and rearranging the above equation yield

$$
C_{n}^{b}=\left[\begin{array}{lll}
C_{11} & C_{12} & C_{13} \\
C_{21} & C_{22} & C_{23} \\
C_{31} & C_{32} & C_{33}
\end{array}\right]=\left[\begin{array}{ccc}
\cos \gamma \cos \psi+\sin \gamma \sin \theta \sin \psi & -\cos \gamma \sin \psi+\sin \gamma \sin \theta \cos \psi & -\sin \gamma \cos \theta \\
\cos \theta \sin \psi & \cos \theta \cos \psi & \sin \theta \\
\sin \gamma \cos \psi-\cos \gamma \sin \theta \sin \psi & -\sin \gamma \sin \psi-\cos \gamma \sin \theta \cos \psi & \cos \gamma \cos \theta
\end{array}\right] .
$$

The purpose of attitude solution is to figure out these three attitude angles [14].

In $b$-frame, axes $o x_{b}, o y_{b}$ are parallel to the plane of the rotating platform with axis $o z_{b}$ along the rotation axis of the platform. An inertial measurement unit (IMU) generally consists of three mutually perpendicular gyroscopes and accelerometers to measure the angular rates and linear accelerations, respectively. The IMU is mounted on the rotating platform, and the axes $o x_{a}, o y_{a}$ in $a$-frame are parallel to the plane of the rotating platform. The axis $o z_{a}$ lies along the direction of the rotation axis $o z_{b}$, with axis $o x_{a}$ coinciding with axis $o x_{b}$ before rotating. In order to provide the three orthogonal gyroscopes with the same biasing rate, the angular rate of the rotating platform should satisfy the following:

$$
\begin{gathered}
\left|\omega_{x}^{g}\right|^{2}+\left|\omega_{y}^{g}\right|^{2}+\left|\omega_{z}^{g}\right|^{2}=\left|\omega_{z}^{r}\right|^{2}, \\
\left|\omega_{x}^{g}\right|=\left|\omega_{y}^{g}\right|=\left|\omega_{z}^{g}\right| .
\end{gathered}
$$

Then the following is obtained:

$$
\begin{aligned}
& \left|\omega_{x}^{g}\right|=\left|\omega_{y}^{g}\right|=\left|\omega_{z}^{g}\right|=\omega_{\text {bias }}=\frac{1}{\sqrt{3}}\left|\omega_{z}^{\mathrm{r}}\right|, \\
& \chi_{1}=\chi_{2}=\chi_{3}=\arctan \left(\frac{1}{\sqrt{3}}\right)=54.73^{\circ} .
\end{aligned}
$$

So this configuration has each of the three sensitive axes of mutually perpendicular gyroscopes at $54.73^{\circ}$ to the rotation axis $o z_{b}\left(o z_{a}\right)$ and is equally spaced at $120^{\circ}$ to each other in the plane of the rotating platform [16]. This system is rotated away from axis $o z_{b}\left(o z_{a}\right)$, so the three gyroscopes are provided $1 / \sqrt{3}$ of the rotating rate $\omega_{0}$. Meanwhile ensuring that axes $o x_{a}, o x_{g}$, and $o z_{b}\left(o z_{a}\right)$ are in the same plane, the orthogonal instrument cluster arrangement structure relationship between $b$-frame coordinate, $g$-frame coordinate, and $a$-frame coordinate is as shown in Figure 3. 


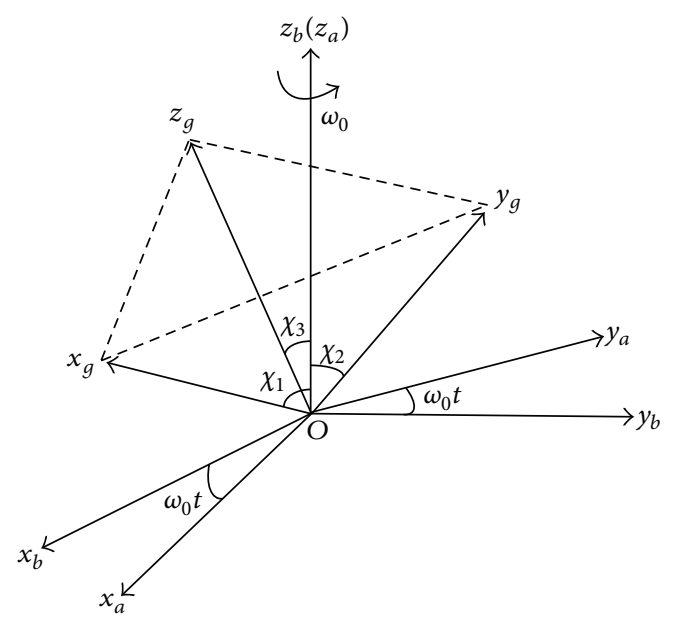

FIGURE 3: The schematic diagram of inertial measurement unit.

Fixed to the rotating platform and the zero-angle position of the rotating platform lying along the vehicle longitudinal axis, the IMU provides measurement of angular rates and linear accelerations about three orthogonal axes. In the presence of continuous rotation at constant rate, IMU dynamic output can be represented by $[14,15]$

$$
\begin{gathered}
f^{a}=C_{b}^{a} C_{n}^{b} f^{n}+n^{a}(t)+b^{a} \\
\omega_{i g}^{g}=C_{a}^{g}\left[C_{b}^{a} C_{n}^{b}\left(\omega_{i e}^{n}+\omega_{e n}^{n}\right)+C_{b}^{a} \omega_{n b}^{b}+\omega_{b a}^{a}\right] \\
+\omega_{a g}^{g}+n^{g}(t)+b^{g},
\end{gathered}
$$

where $f^{a}$ is the measured linear acceleration in $a$-frame; $\omega_{i g}^{g}$ is the measured angular rate in $g$-frame. $n^{a}(t), b^{a}$ are the random drift noise and constant drift noise of the accelerator, respectively. $n^{g}(t), b^{g}$ are the random drift noise and constant drift noise of the gyroscope respectively. It is assumed that the vehicle is stationary relative to the Earth, so $\omega_{e n}^{n}$ and $\omega_{n b}^{n}$ are zero. Also there is no relative movement between $a$-frame and $g$-frame, which means $\omega_{a g}^{g}=0$. Equation (7) could be simplified as

$$
\begin{gathered}
f^{a}=C_{b}^{s} C_{n}^{b} f^{n}+n^{a}(t)+b^{a}, \\
\omega_{i g}^{g}=C_{a}^{g}\left(C_{b}^{a} C_{n}^{b} \omega_{i e}^{n}+\omega_{b a}^{a}\right)+n^{g}(t)+b^{g} .
\end{gathered}
$$

Ignoring scale-factor error and installation error of the inertial component and substituting the above coefficients, (8) can be further written as (9) and (11).

As discussed above, in the presence of continuous and unidirectional rotation with constant rate, the specific forces acting on the vehicle, measured by three mutually perpendicular accelerometers, are given by

$$
\begin{aligned}
{\left[\begin{array}{l}
F_{x}^{a} \\
F_{y}^{a} \\
F_{z}^{a}
\end{array}\right]=} & {\left[\begin{array}{ccc}
\cos \left(\omega_{0} t\right) & \sin \left(\omega_{0} t\right) & 0 \\
-\sin \left(\omega_{0} t\right) & \cos \left(\omega_{0} t\right) & 0 \\
0 & 0 & 1
\end{array}\right]\left[\begin{array}{lll}
C_{11} & C_{12} & C_{13} \\
C_{21} & C_{22} & C_{23} \\
C_{31} & C_{32} & C_{33}
\end{array}\right]\left[\begin{array}{l}
0 \\
0 \\
g
\end{array}\right] } \\
& +\left[\begin{array}{l}
n_{x}^{a}(t) \\
n_{y}^{a}(t) \\
n_{z}^{a}(t)
\end{array}\right]+\left[\begin{array}{l}
b_{x}^{a} \\
b_{y}^{a} \\
b_{z}^{a}
\end{array}\right] .
\end{aligned}
$$

This equation can be rearranged and expressed as follows:

$$
\left[\begin{array}{c}
F_{x}^{a} \\
F_{y}^{a} \\
F_{z}^{a}
\end{array}\right]=\left[\begin{array}{c}
C_{13} g \cos \left(\omega_{0} t\right)-C_{23} g \sin \left(\omega_{0} t\right)+n_{x}^{a}(t)+b_{x}^{a} \\
C_{13} g \sin \left(\omega_{0} t\right)+C_{23} g \cos \left(\omega_{0} t\right)+n_{y}^{a}(t)+b_{y}^{a} \\
C_{33} g+n_{z}^{a}(t)+b_{z}^{a}
\end{array}\right] .
$$

As could be seen from (10), the outputs of the three accelerometers involve $C_{13}, C_{23}$, and $C_{33}$ of the attitude matrix $C_{n}^{b}$ in (4), which are only associated with the horizontal attitude angles $\gamma, \theta$. It will be seen that the specific forces $F_{x}^{a}$ and $F_{y}^{a}$ are expressed in the form of sine and cosine waveforms with the same amplitudes but different phases. Similarly, ignoring the random drift noise, the output of the accelerometer $F_{z}^{a}$ is approximated as a constant value.

Meanwhile, the angular rates sensed by three orthogonal gyroscopes sensitive axes are given by

$$
\begin{aligned}
& {\left[\begin{array}{l}
G_{x}^{g} \\
G_{y}^{g} \\
G_{z}^{g}
\end{array}\right]=\left[\begin{array}{ccc}
\frac{2}{\sqrt{6}} & 0 & \frac{1}{\sqrt{3}} \\
-\frac{1}{\sqrt{6}} & \frac{1}{\sqrt{2}} & \frac{1}{\sqrt{3}} \\
-\frac{1}{\sqrt{6}} & -\frac{1}{\sqrt{2}} & \frac{1}{\sqrt{3}}
\end{array}\right]} \\
& \times\left(\left[\begin{array}{ccc}
\cos \left(\omega_{0} t\right) & \sin \left(\omega_{0} t\right) & 0 \\
-\sin \left(\omega_{0} t\right) & \cos \left(\omega_{0} t\right) & 0 \\
0 & 0 & 1
\end{array}\right]\left[\begin{array}{lll}
C_{11} & C_{12} & C_{13} \\
C_{21} & C_{22} & C_{23} \\
C_{31} & C_{32} & C_{33}
\end{array}\right]\right. \\
& \left.\times\left[\begin{array}{c}
0 \\
\omega_{i e N}^{n} \\
\omega_{i e U}^{n}
\end{array}\right]-\left[\begin{array}{c}
0 \\
0 \\
\omega_{0}
\end{array}\right]\right) \\
& +\left[\begin{array}{l}
n_{x}^{g}(t) \\
n_{y}^{g}(t) \\
n_{z}^{g}(t)
\end{array}\right]+\left[\begin{array}{l}
b_{x}^{g} \\
b_{y}^{g} \\
b_{z}^{g}
\end{array}\right] \text {. }
\end{aligned}
$$


The above equation can be rearranged and expressed as follows:

$$
\left[\begin{array}{l}
G_{x}^{g} \\
G_{y}^{g} \\
G_{z}^{g}
\end{array}\right]=\left[\begin{array}{c}
\frac{2 R}{\sqrt{6}} \cos \left(\omega_{0} t\right)+\frac{2 M}{\sqrt{6}} \sin \left(\omega_{0} t\right)+\frac{N-\omega_{0}}{\sqrt{3}}+n_{x}^{g}(t)+b_{x}^{g} \\
\frac{\sqrt{3} M-R}{\sqrt{6}} \cos \left(\omega_{0} t\right)-\frac{M+\sqrt{3} R}{\sqrt{6}} \sin \left(\omega_{0} t\right)+\frac{N-\omega_{0}}{\sqrt{3}}+n_{y}^{g}(t)+b_{y}^{g} \\
-\frac{\sqrt{3} M+R}{\sqrt{6}} \cos \left(\omega_{0} t\right)-\frac{M-\sqrt{3} R}{\sqrt{6}} \sin \left(\omega_{0} t\right)+\frac{N-\omega_{0}}{\sqrt{3}}+n_{z}^{g}(t)+b_{z}^{g}
\end{array}\right],
$$

where $R=C_{12} \omega_{i e N}^{n}+C_{13} \omega_{i e U}^{n}, M=C_{22} \omega_{i e N}^{n}+$ $C_{23} \omega_{i e U}^{n}$, and $N=C_{32} \omega_{i e N}^{n}+C_{33} \omega_{i e U}^{n}$.

According to (12), the output of a triad of gyroscopes is related to both the heading angle $\psi$ and horizontal attitude angles $\gamma, \theta$, which comprise sinusoidal oscillation superimposed on the constant parameter $\left(N-\omega_{0}\right) / \sqrt{3}$. Clearly, it biases the measurement signals of the RLG away from the lock-in region, reducing the measurement errors.

As mentioned above, the rotating rate modulates the stationary attitude signals as sinusoidal signals, which facilitates the subsequent process of attitude solution. According to the characteristic of the output signals, the acceleration outputs $F_{x}^{a}, F_{y}^{a}$ only contain the horizontal attitude signals, and the gyroscope outputs $G_{x}^{g}, G_{y}^{g}$, and $G_{z}^{g}$ contain all the attitude signals. Since the outputs of the inertial components have similar propagation rule, the attitude angles could be determined from each combination outputs of $F_{x}^{a}-G_{x}^{g}, F_{x}^{a}$ $G_{y}^{g}, F_{x}^{a}-G_{z}^{g}, F_{y^{-}}^{a} G_{x}^{g}, F_{y^{-}}^{a} G_{y}^{g}$, and $F_{y^{-}}^{a} G_{z}^{g}$, which are six simplified configurations in total. The solution of horizontal attitude angles is the premise to calculate the heading angle.

\section{The Proposed Algorithm for North Finding}

3.1. The Principle of the Lock-In Amplifier. Lock-in amplifier (LIA) was firstly used to suppress $1 / \mathrm{f}$ noise and constant drift induced by the thermal effects of the circuits and to extract weak signal from those with strong noises [7$10]$. It is also widely employed in resource exploration and precision measurement field, and better results are obtained especially for the sine waves and square waves. According to the characteristic of the measurement signal $x(t)$, the lockin amplifier generates two orthogonal reference signals $r_{I}(t)$ and $r_{Q}(t)$, the frequencies of which are consistent with $x(t)$. These two reference signals have the same amplitude but $90^{\circ}$ phase difference. The spectrum of the measurement signal $x(t)$ could be changed by multiplication and accumulation operations of two reference signals $r_{I}(t)$ and $r_{Q}(t)$ in the phase sensitive devise, and then the drift noise in the signal is removed through a low-pass filter consequently. Since the output signal of LIA is sensitive to the amplitude and phase of the measurement signal $x(t)$, both amplitude discriminating and phase discriminating are accomplished to extract the feature parameters (amplitude and phase) of the measurement signal, as shown in Figure 4.
In Figure 4, the single frequency measurement signal $x(t)$ without drift noise can be represented by $V_{s} \sin \left(\omega_{0} t+\eta\right)$. The two square waves reference signals are $r_{I}(t), r_{Q}(t)$ with the same amplitude $V_{r}$ and $90^{\circ}$ phase-difference. After the signal sampling with integral multiple of rotation periods, In-phase $(I)$ and Quadrature $(Q)$ components are calculated:

$$
\begin{aligned}
& I=\frac{1}{t} \sum_{0}^{t} x(t) \cdot r_{I}(t)=\frac{2}{\pi} V_{s} V_{r} \cos \eta, \\
& Q=\frac{1}{t} \sum_{0}^{t} x(t) \cdot r_{Q}(t)=\frac{2}{\pi} V_{s} V_{r} \sin \eta .
\end{aligned}
$$

The following are obtained:

$$
\begin{gathered}
V_{s}=\frac{\pi}{2 V_{r}} \sqrt{I^{2}+Q^{2}}, \\
\eta=\arctan \left(\frac{Q}{I}\right) .
\end{gathered}
$$

As mentioned above, the feature parameters (amplitude $V_{s}$ and phase $\eta$ ) of the sinusoidal measurement signal could be identified by the lock-in amplifier [10].

3.2. The Attitude Solution Algorithm Proposed for the North Finder. As a result of the preponderance of trigonometric terms in (10) and (12), the accelerometer signals only contain the horizontal attitude signals. The amplitude and phase of the measurement signal could be extracted by the lockin amplifier, allowing two horizontal attitude angles to be obtained by resolving the accelerometer measurements. Similarly, the heading angle could be obtained by resolving gyroscope measurement signal through another lock-in amplifier consequently according to (13) and (14).

In practice, the rotation axis should be kept perpendicular to the vehicle base so that the rolling back and forth motion of accelerometer in the gravitational field could be avoided effectively, and the accelerometer works in linear measurement region.

For the sake of easy installation, a simplified configuration of north finder system is proposed. The system mainly consists of $o x_{a}$-axis accelerometer, $o x_{q}$-axis laser gyroscope, and a single-axis rotating platform. $F_{x}^{a}$ and $G_{x}^{g}$ are the $o x_{a^{-}}$ axis accelerometer output signal and $o x_{g}$-axis gyroscope 
output signal, respectively, which are also used as the measurement signals of the lock-in amplifiers.

In terms of the components $C_{13}$ and $C_{23}$ of the unknown matrix $C_{n}^{b}$, the amplitude $V_{s}$ and the phase $\eta$ of the $o x_{a}$-axis accelerometer signal $F_{x}^{a}$ could be written as

$$
\begin{aligned}
F_{x}^{a} & =C_{13} g \cos \left(\omega_{0} t\right)-C_{23} g \sin \left(\omega_{0} t\right)+n_{x}^{a}(t)+b_{x}^{a} \\
& =\sqrt{C_{13}^{2}+C_{23}^{2}} g \sin \left(\omega_{0} t-\arctan \frac{C_{13}}{C_{23}}\right)+n_{x}^{a}(t)+b_{x}^{a} .
\end{aligned}
$$

Due to the presence of the random drift noise, the amplitude and phase calculated by the lock-in amplifier have some deviations from the true values, shown as

$$
\begin{gathered}
\widehat{V}_{S}=\sqrt{C_{13}^{2}+C_{23}^{2}} g, \\
\widehat{\eta}=-\arctan \frac{C_{13}}{C_{23}} .
\end{gathered}
$$

Combining (4), (14), and (16), the amplitude and phase of the signal $F_{x}^{a}$ from the accelerometer could be calculated, and then $C_{13}$ and $C_{23}$ are determined. Since the inverse-sine function is monotonic in the main interval $\left[-90^{\circ}, 90^{\circ}\right]$, the horizontal attitude angles $\theta$ and $\gamma$ could be determined solely from $C_{13}$ and $C_{23}$, as shown in

$$
\begin{gathered}
\theta=\theta_{\text {main }}=\sin ^{-1}\left(C_{23}\right), \\
\gamma=\gamma_{\text {main }}=\sin ^{-1}\left(-\frac{C_{13}}{\cos \theta}\right),
\end{gathered}
$$

where $\theta_{\text {main }}$ and $\gamma_{\text {main }}$ are the main values of the $\theta$ and $\gamma$ in the inverse-sine function.

Similarly, The output of the $o x_{g}$-axis laser gyroscope signal $G_{x}^{g}$ is expressed as

$$
\begin{aligned}
G_{x}^{g}= & \frac{2 R}{\sqrt{6}} \cos \left(\omega_{0} t\right)+\frac{2 M}{\sqrt{6}} \sin \left(\omega_{0} t\right)+\frac{N-\omega_{0}}{\sqrt{3}}+b_{x}^{g}+n_{x}^{g}(t) \\
= & \frac{2 \sqrt{M^{2}+R^{2}}}{\sqrt{6}} \sin \left(\omega_{0} t+\arctan \frac{R}{M}\right) \\
& +\frac{N-\omega_{0}}{\sqrt{3}}+b_{x}^{g}+n_{x}^{g}(t),
\end{aligned}
$$

where $R=C_{12} \omega_{i e N}^{n}+C_{13} \omega_{i e U}^{n}, M=C_{22} \omega_{i e N}^{n}+C_{23} \omega_{i e U}^{n}$, and $N=C_{32} \omega_{i e N}^{n}+C_{33} \omega_{i e U}^{n}$.

And $\omega_{i e E}^{n}=0, \omega_{i e N}^{n}=\omega_{i e}^{n} \cdot \cos L$, and $\omega_{i e U}^{n}=\omega_{i e}^{n} \cdot \sin L$ are the Earth's rate components which could be calculated from the known local latitude $L$. Thus, parameters $M, R$ could be expressed as

$$
\begin{aligned}
M= & C_{22} \cdot \omega_{i e N}^{n}+C_{23} \cdot \omega_{i e U}^{n} \\
= & \cos \theta \cos \psi \cdot \omega_{i e N}^{n}+\sin \theta \cdot \omega_{i e U}^{n}, \\
R= & C_{12} \cdot \omega_{i e N}^{n}+C_{13} \cdot \omega_{i e U}^{n} \\
= & (-\cos \gamma \sin \psi+\sin \gamma \sin \theta \cos \psi) \cdot \omega_{i e N}^{n} \\
& -\sin \gamma \cos \theta \cdot \omega_{i e U}^{n} .
\end{aligned}
$$

TABLE 1: The truth value table of the heading angle $\psi$.

\begin{tabular}{lcc}
\hline $\cos \psi$ & $\sin \psi$ & Heading angle $\psi$ \\
\hline+ & + & $\psi_{\text {main }}$ \\
- & + & $\psi_{\text {main }}+180^{\circ}$ \\
- & - & $\psi_{\text {main }}+180^{\circ}$ \\
+ & - & $\psi_{\text {main }}+360^{\circ}$ \\
1 & 0 & $0^{\circ}$ \\
0 & 1 & $90^{\circ}$ \\
-1 & 0 & $180^{\circ}$ \\
0 & -1 & $270^{\circ}$ \\
\hline
\end{tabular}

The main value of heading angle $\psi_{\text {main }}$ could be computed as follows:

$$
\cos \psi=\frac{\left(M-\sin \theta \cdot \omega_{i e U}^{n}\right)}{\left(\cos \theta \cdot \omega_{i e N}^{n}\right)},
$$

$$
\begin{gathered}
\sin \psi=\frac{\left[\sin \gamma \tan \theta \cdot\left(M-\sin \theta \cdot \omega_{i e U}^{n}\right)-\sin \gamma \cos \theta \cdot \omega_{i e U}^{n}-R\right]}{\left(\cos \gamma \cdot \omega_{i e N}^{n}\right)}, \\
\psi_{\text {main }}=\arctan \left(\frac{\sin \psi}{\cos \psi}\right), \quad \text { where } \psi_{\text {main }} \in\left(-90^{\circ}, 90^{\circ}\right) .
\end{gathered}
$$

As the heading angle $\psi$ lies in the interval $\left[0^{\circ}, 360^{\circ}\right)$, it could be calculated through $\psi_{\text {main }}$ in (20), finishing the whole process of north finding. The truth table of the heading angle $\psi$ is shown in Table 1 .

It is shown that the range of heading angle $\psi$ is $\psi \epsilon$ $\left[0^{\circ}, 360^{\circ}\right)$, and the range of main value $\psi_{\text {main }}$ is $\psi_{\text {main }} \epsilon$ $\left(-90^{\circ}, 90^{\circ}\right)$. There are totally eight cases for the heading angle $\psi$, according to the signs of $\cos \psi, \sin \psi$.

\subsection{Determination of the Inertial Components Constant Drift} Noise. As shown in Figure 5, the outputs of the accelerometer $F_{x}^{a}$ and gyroscope $G_{x}^{a}$ are sinusoidal oscillatory motions with equal frequency summed by a constant parameter. The constant drift noises of the inertial components are estimated by averaging the data in an entire rotation period, which can eliminate the influence of sinusoidal oscillation. The estimations of the constant drift noises of the inertial components are mainly affected by the intensity of random noise and the stability of the rotating rate. Combining (10), (12) and ignoring the random drift noise, the expressions for constant drift noises of accelerometer and gyroscope are given as

$$
\begin{aligned}
b_{x}^{a} & \approx \frac{1}{t} \sum_{0}^{t}\left[C_{13} g \cos \left(\omega_{0} t\right)-C_{23} g \sin \left(\omega_{0} t\right)+n_{x}^{a}(t)\right] \\
& =\frac{1}{t} \sum_{0}^{t} F_{x}^{a}, \\
b_{x}^{g} & \approx \frac{1}{t} \sum_{0}^{t}\left[\frac{2 M}{\sqrt{6}} \sin \left(\omega_{0} t\right)+\frac{2 R}{\sqrt{6}} \cos \left(\omega_{0} t\right)+n_{x}^{g}(t)\right]
\end{aligned}
$$




$$
\begin{aligned}
= & \frac{1}{t} \sum_{0}^{t} G_{x}^{g}-\frac{N-\omega_{0}}{\sqrt{3}}, \\
N= & C_{32} \omega_{i e N}^{n}+C_{33} \omega_{i e U}^{n} \\
= & -\sin \gamma \sin \psi \cdot \omega_{i e N}^{n}-\cos \gamma \sin \theta \cos \psi \cdot \omega_{i e N}^{n} \\
& +\cos \gamma \sin \theta \cdot \omega_{i e U}^{n},
\end{aligned}
$$

where $t=n T_{0}, n \in N^{+} . T_{0}$ is the time period for rotating one circle $\left(360^{\circ}\right)$, and $t$ is the time for $n$ circles of the platform rotation.

\section{Configuration and Experiment of the North Finder}

4.1. Configuration of the North Finder System. All of the components of the north finder system are fixed on the base of the vehicle, and the plane of rotating platform is parallel to the plane of the vehicle base. Make sure that the sensitive axis of the accelerometer, the sensitive axis of the laser gyroscope, and the rotation axis lie in the same plane, and the sensitive axis of the gyroscope forms an angle $\chi_{1}$ with the rotation-axis for the equipment configuration considered above. The skewed sensor configuration ensures that the rotating platform provides the gyroscope with a biasing frequency to overcome the lock-in phenomenon. The zeroangle position of the rotating platform is usually arranged to be aligned with the longitudinal axis of the vehicle; then the heading angle could be determined by combining the outputs from the inertial components and angle encoders [14]. The structure diagram of the north finder configuration system is shown in Figure 5.

Since the rotating platform continuously rotates at a constant rate, a conductive ring is required to connect the signal line and power line. An accurate controller of rotating rate with the compensation for friction torque is critical to the accurate estimation of heading angle [17]. To guarantee stability of rotating rate, a bipolar PWM control mode is employed in the servo system, and Phase Locked Loop (PLL) technology is also used, which comprises four parts: the control algorithm, a frequency discriminator, a low-pass filter, and power driver [18-20]. The angle between rotating platform and the base is measured by an angle encoder with arc-second accuracy, the output of which would be feed back via torque motor to rotate the platform at the reference rate provided. The rotating platform can be also oriented at the zero-angle position at initial time automatically.

Two orthogonal square waves with $90^{\circ}$ out of phase are adopted as the reference signals. When the platform rotates at the instant that the output of angle encoder is an integer multiple of $90^{\circ}$, interrupts are triggered to switch to both polarities of the reference signals, respectively. The data from inertial components are not collected until a steady motor rate is reached. After data collection, the two horizontal attitude angles could be calculated from the accelerometer output $F_{x}^{a}$ by the LIA1; then the heading angle is determined
TABLE 2: Typical performance parameters for the inertial components.

\begin{tabular}{lc}
\hline Sources of errors & Values \\
\hline The constant drift noise of the gyroscope $\left(b^{g}\right)$ & $0.02^{\circ} / \mathrm{h}$ \\
The random drift noise of the gyroscope $\left(n^{g}(t)\right)$ & $0.01^{\circ} / \mathrm{h}$ \\
The installation error of the gyroscope $\left(c^{g}\right)$ & $10^{\prime \prime}$ \\
The scale-factor error of the gyroscope $\left(s^{g}\right)$ & $10 \mathrm{ppm}$ \\
The constant drift noise of the accelerometer $\left(b^{a}\right)$ & $100 \mu \mathrm{g}$ \\
The random drift noise of the accelerometer $\left(n^{a}(t)\right)$ & $50 \mu \mathrm{g}$ \\
The installation error of the accelerometer $\left(c^{a}\right)$ & $10^{\prime \prime}$ \\
The scale-factor error of the accelerometer $\left(s^{a}\right)$ & $10 \mathrm{ppm}$ \\
\hline
\end{tabular}

TABLE 3: Experimental data processing results of ten groups repeated north finding.

\begin{tabular}{lccc}
\hline Experimental groups & \multicolumn{3}{c}{ The vehicle's attitudes } \\
& $11.9922^{\circ}$ & $-14.9921^{\circ}$ & $276.0142^{\circ}$ \\
\hline Group 1 results & $11.9923^{\circ}$ & $-14.9920^{\circ}$ & $276.0127^{\circ}$ \\
Group 2 results & $11.9923^{\circ}$ & $-14.9922^{\circ}$ & $276.0163^{\circ}$ \\
Group 3 results & $11.9922^{\circ}$ & $-14.9920^{\circ}$ & $276.0162^{\circ}$ \\
Group 4 results & $11.9924^{\circ}$ & $-14.9921^{\circ}$ & $276.0155^{\circ}$ \\
Group 5 results & $11.9923^{\circ}$ & $-14.9920^{\circ}$ & $276.0152^{\circ}$ \\
Group 6 results & $11.9923^{\circ}$ & $-14.9920^{\circ}$ & $276.0162^{\circ}$ \\
Group 7 results & $11.9922^{\circ}$ & $-14.9921^{\circ}$ & $276.0134^{\circ}$ \\
Group 8 results & $11.9923^{\circ}$ & $-14.9920^{\circ}$ & $276.0145^{\circ}$ \\
Group 9 results & $11.9923^{\circ}$ & $-14.9921^{\circ}$ & $276.0157^{\circ}$ \\
Group 10 results & $11.9923^{\circ}$ & $-14.9921^{\circ}$ & $276.0150^{\circ}$ \\
The mean of the & & & \\
attitude angles & & & \\
The maximum of the & $0.0163^{\circ}\left(0.978^{\prime}\right)$ & & \\
heading error & & & \\
\hline
\end{tabular}

from the gyroscope output $G_{x}^{g}$ by the LIA2, the principle diagram of which is shown in Figure 6.

4.2. Simulation Experiment. Under static condition, the vehicle's attitudes $[\gamma \theta \psi]$ are $\left[12^{\circ}-15^{\circ} 276^{\circ}\right]$, and the skewed angle $\chi_{1}$ is $54.73^{\circ}$. Each sensor output is sampled at a rate of $100 \mathrm{~Hz}$, with the rotating rate $\omega_{0}=10^{\circ} / \mathrm{s}$ and the operation time $t=72 \mathrm{~s}$. According to the proposed principle, a simulation experiment is designed with the typical performance parameters for the inertial components shown in Table 2.

Ten groups of north finding experiments are executed, and data processing results are shown in Table 3.

It can be seen that the accuracy of horizontal attitude angles $\gamma, \theta$ is higher than that of heading angle $\psi$ from the above ten groups' results. Simulation data results show that the maximum heading error is less than $1.0^{\prime}$ in $72 \mathrm{~s}$, when the rotating rate is $\omega_{0}=10^{\circ} / \mathrm{s}$.

\subsection{Error Analysis}

4.3.1. Effect of Drift Noise on the Accuracy of the North Finding. Uniformity effects on drift noise can be very significant either 


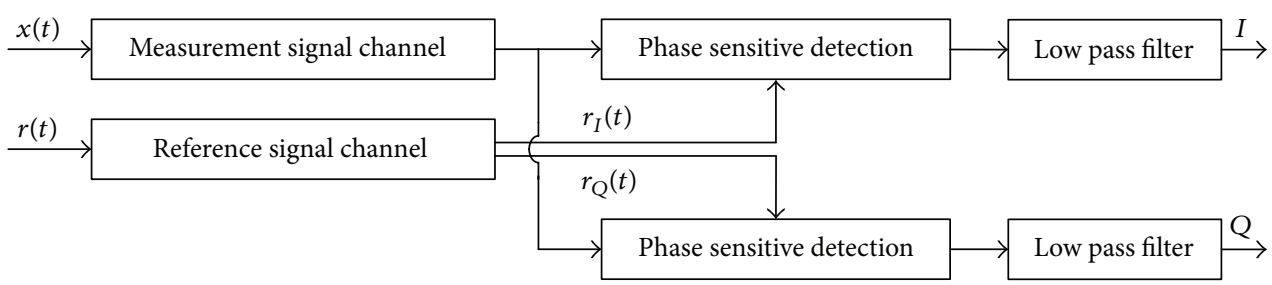

FIGURE 4: Lock-in amplifier structure figure.

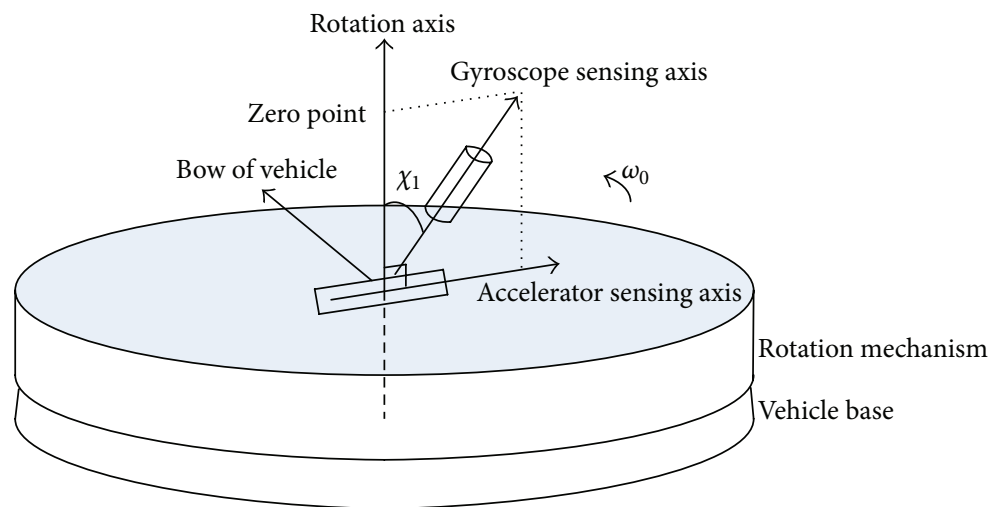

Figure 5: The structure diagram of the north finder.

optically or electronically, which is often modeled as additive Gaussian white noise and constant noise $[11,21]$. The output of the inertial component is given by

$$
x(t)=V_{s} \sin \left(\omega_{0} t+\eta\right)+n(t)+b,
$$

where $n(t)$ and $b$ represent the random drift noise and constant drift noise, respectively. Taking the wideband drift noise $n(t)$ into account, (22) can be rewritten as

$$
x(t)=V_{s} \sin \left(\omega_{0} t+\eta\right)+\sum_{n=1}^{\infty} V_{n} \sin \left(\omega_{n} t+\eta_{n}\right)+b,
$$

where $V_{s}$ is the amplitude of the measurement signal, $V_{n}$ and $\eta_{n}$ are the amplitude and phase of the random drift noise $n(t)$ in each frequency, respectively.

One of the reference signals can be expanded into the Fourier series [22]:

$$
r(t)=\frac{4 V_{r}}{\pi} \sum_{n=1}^{\infty} \frac{(-1)^{n+1}}{2 n-1} \cos \left[(2 n-1) \omega_{0} t\right] .
$$

The product of $x(t)$ by $r(t)$ yields

$$
\begin{aligned}
u_{\mathrm{out}} & =x(t) \times r(t) \\
& =\frac{2 V_{s} V_{r}}{\pi} \sum_{n=1}^{\infty} \frac{(-1)^{n+1}}{2 n-1} \sin \left[(2 n-2) \omega_{0} t-\eta\right]
\end{aligned}
$$

$$
\begin{aligned}
& +\frac{2 V_{s} V_{r}}{\pi} \sum_{n=1}^{\infty} \frac{(-1)^{n+1}}{2 n-1} \sin \left(2 n \omega_{0} t+\eta\right) \\
& +\frac{2 V_{s} V_{r}}{\pi} \sum_{n=1}^{\infty} \frac{(-1)^{n+1}}{2 n-1} \sin \left[\left(\omega_{n}+(2 n-1) \omega_{0}\right) t+\eta_{n}\right] \\
& +\frac{2 V_{s} V_{r}}{\pi} \sum_{n=1}^{\infty} \frac{(-1)^{n+1}}{2 n-1} \sin \left[\left(\omega_{n}-(2 n-1) \omega_{0}\right) t+\eta_{n}\right] \\
& +\frac{4 b V_{r}}{\pi} \sum_{n=1}^{\infty} \frac{(-1)^{n+1}}{2 n-1} \cos \left[(2 n-1) \omega_{0} t\right]
\end{aligned}
$$

As shown in (25), the product $u_{\text {out }}$ consists of five components: only $n=1$ frequency difference has an output after the low-pass filter in the first term, which is a useful information to obtain the amplitude $V_{s}$ and phase $\eta$ of the measurement signal $x(t)$. Other frequency components in the first term, the second term, and the third term can be filtered out by the low-pass filter. There is no frequency difference in the fifth term, allowing no output through the low-pass filter, so the output is not impacted by the constant drift noise $b$; most of the frequency components of wideband random drift noise $n(t)$ can be filtered out in the fourth term, but when the frequency of the drift noise and the corresponding harmonic frequency of the reference square wave have the relationship $\omega_{n}=(2 n-1) \omega_{0}$, a direct current output of a phase sensitive is produced, which influences the result to some extent. Therefore, in order to eliminate the effect of the harmonics, a band-pass filter with the center frequency $\omega_{0}$ is added to filter out the higher harmonic in 


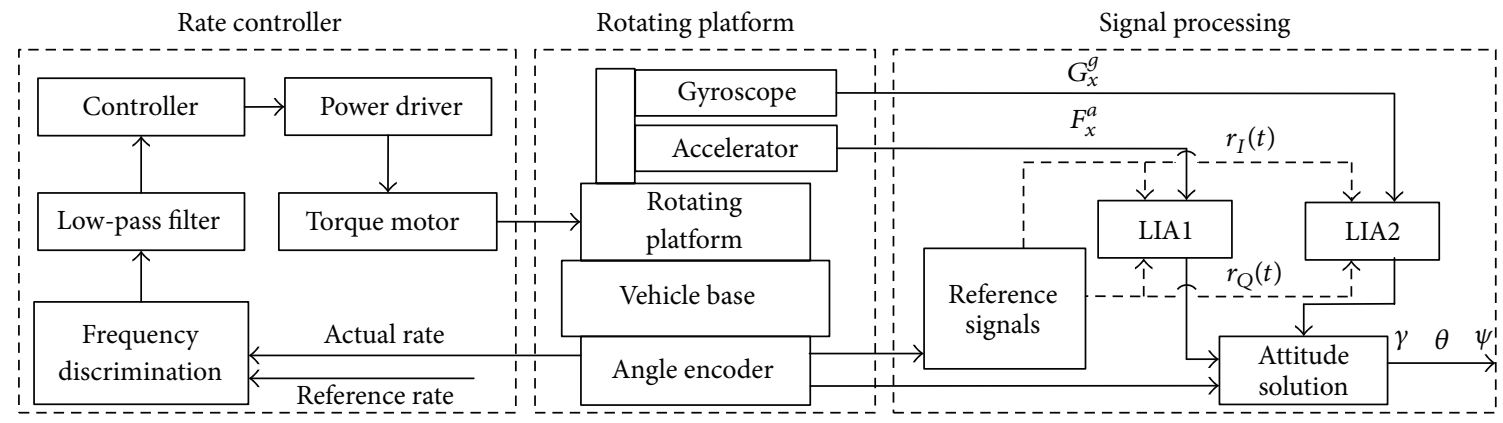

FIgURE 6: The principle diagram of the north finder.

the measurement signal channel, using the function of its narrowband characteristic.

\subsubsection{Effect of Rate Stability on the Accuracy of the North} Finding. Here, the drift noises of the inertial components are ignored, and only the deviation of rotating rate is considered [2]. Thus the constant rate $\omega_{0}$ has a random frequency component, which randomizes slightly the angular motion of the rotating platform [23]. Therefore, the output signal of the inertial measurement is given by

$$
\begin{aligned}
x(t)=V_{s} & \sin \left[\omega_{0} t+\eta+\theta_{0} \sin (\alpha t)\right] \\
=V_{s} & \sin \left(\omega_{0} t+\eta\right) \cos \left(\theta_{0} \sin \alpha t\right) \\
& \left.\quad+\cos \left(\omega_{0} t+\eta\right) \sin \left(\theta_{0} \sin \alpha t\right)\right] .
\end{aligned}
$$

Expanding $\cos \left(\theta_{0} \sin \alpha t\right)$ and $\sin \left(\theta_{0} \sin \alpha t\right)$ into the form of the first Bessel function yields

$$
\begin{aligned}
x(t)= & V_{s} J_{0} \sin \left(\omega_{0} t+\eta\right)+V_{s} \sum_{m=1}^{\infty} J_{m}\left[\sin \left(\left(\omega_{0}+m a\right) t+\eta\right)\right] \\
& +(-1)^{m} \sin \left(\left(\omega_{0}-m a\right) t+\eta\right]
\end{aligned}
$$

where $J_{m}$ is the coefficient of the first Bessel function, $m$ is the order, and $\theta_{0}$ is the amplitude of the frequency error. As $\theta_{0}$ is very small, $J_{1}=\theta_{0} / 2, J_{2}=J_{3}=J_{4} \cdots \approx 0$, and (27) could be rewritten as

$$
x(t)=V_{s} \sin \left(\omega_{0} t+\eta\right)+V_{s} \theta_{0} \cos \left(\omega_{0} t+\eta\right) .
$$

One of the reference signals can be expanded into the Fourier series [22]:

$$
r(t)=\frac{4 V_{r}}{\pi} \sum_{n=1}^{\infty} \frac{(-1)^{n+1}}{2 n-1} \cos \left[(2 n-1) \omega_{0} t\right] .
$$

According to (28) and (29), the product of $x(t)$ by $r(t)$ yields:

$$
\begin{aligned}
u_{\mathrm{out}}= & x(t) \times r(t) \\
= & \frac{2 V_{s} V_{r}}{\pi} \sum_{n=1}^{\infty} \frac{(-1)^{n+1}}{2 n-1} \sin \left[(2 n-2) \omega_{0} t-\eta\right] \\
& +\frac{2 V_{s} V_{r}}{\pi} \sum_{n=1}^{\infty} \frac{(-1)^{n+1}}{2 n-1} \sin \left(2 n \omega_{0} t+\eta\right) \\
& +\frac{\theta_{0} V_{s} V_{r}}{\pi} \sum_{n=1}^{\infty} \frac{(-1)^{n+1}}{2 n-1} \sin \left[(2 n-2) \omega_{0} t-\alpha t-\eta\right] \\
& +\frac{\theta_{0} V_{s} V_{r}}{\pi} \sum_{n=1}^{\infty} \frac{(-1)^{n+1}}{2 n-1} \sin \left(2 n \omega_{0} t+\alpha t+\eta\right) \\
& -\frac{\theta_{0} V_{s} V_{r}}{\pi} \sum_{n=1}^{\infty} \frac{(-1)^{n+1}}{2 n-1} \sin \left[(2 n-2) \omega_{0} t+\alpha t-\eta\right] \\
& -\frac{\theta_{0} V_{s} V_{r}}{\pi} \sum_{n=1}^{\infty} \frac{(-1)^{n+1}}{2 n-1} \sin \left(2 n \omega_{0} t-\alpha t+\eta\right) .
\end{aligned}
$$

As shown in (30), the product $u_{\text {out }}$ consists of six components: only $n=1$ frequency difference has an output after the low-pass filter in the first term, which is considered useful information to obtain the amplitude $V_{s}$ and phase $\eta$ of the measurement signal $x(t)$. Other frequency components in the first term, the second term, the fourth term, and the fifth term can be filtered out by the low-pass filter. The errors of the result are from the frequency-difference of $\alpha=(2 n-2) \omega_{0}$ in the third term, the difference component of $\alpha=2 n \omega_{0}$ in the fifth term and in the sixth term, causing an error output through the lock-in amplifier. Since the amplitude $\theta_{0}$ of the error is small, its effect on the result is not significant.

4.3.3. Effect on North Finder Accuracy due to Installing Error. In the ideal condition, the gyroscope sensitive axis $o x_{g}$ is OAaxis with the skewed angle $\chi_{1}$ to the rotation axis $o z_{b}\left(o z_{a}\right)$. Two parameters of installing error $\partial, \beta$ represent the deviation of the matrix $C_{a}^{g}$ from the ideal condition, and the result of the gyroscope sensitive $o x_{g}^{\prime}$-axis is turned into $O C$-axis. It 


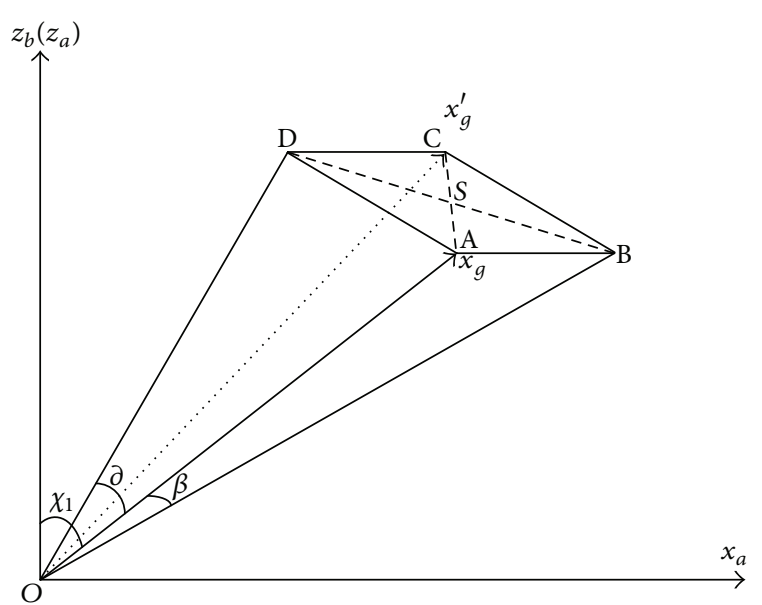

FIGURE 7: Installing error of sensitive axis of the inertial component.

is stressed that, although there is no effect on the output of the $o x_{a}$-axis accelerometer, the angular rate that the $o x_{g}^{\prime}$-axis gyroscope sensed at this time can be expressed as

$$
\begin{aligned}
G_{x}^{g}= & {\left[R \cos \beta \cos \left(\chi_{1}-\partial\right)+M \sin \beta\right] \cos \left(\omega_{0} t\right) } \\
& +\left[M \cos \beta \cos \left(\chi_{1}-\partial\right)-R \sin \beta\right] \sin \left(\omega_{0} t\right) \\
& +\left(N-\omega_{0}\right) \cos \beta \cos \left(\chi_{1}-\partial\right)+b_{x}^{g}+n_{x}^{g}(t) .
\end{aligned}
$$

The horizontal attitude angle solutions will not be affected as the output of the $o x_{a}$-axis accelerometer is not changed by the errors. However, the heading angle solution will be affected. Under the conditions $\partial=12^{\prime \prime}$ and $\beta=12^{\prime \prime}, 15^{\prime \prime}$ heading error will be induced, which illustrates that this type of installation error should be reduced as small as possible [24].

In addition to the installing error described in Figure 7, when the sensitive center of the accelerometer does not go through the center of the rotation axis sufficiently, a steady drift bias of acceleration is introduced as a result of size effect [14]. It does not affect the solution of attitude angles, which is similar to the constant drift noise of the accelerometer.

\section{Conclusion}

This paper focuses on a dynamic strapdown north finding scheme at the constant rate rotation. A simplified configuration of the north finder system consists of three major parts: a pendulous force-feedback accelerometer, an RLG, and a rotating platform, which reduces the cost of equipment. In order to suppress the drift noise of the inertial components, two lock-in amplifiers are employed to extract and amplify the vehicle's attitude signals that are weak and slow-varying. The main advantage of the new scheme keeps the rotating platform away from the process of leveling, which is convenient for engineering applications. The vehicle's attitude angles could be determined in a short time with certain accuracy, as well as the estimations of the constant drift noise of accelerometer and gyroscope.
The only remaining problem of using this scheme arises when the installation errors of gyroscope and accelerometer are significant. These installation errors cause the heading error during north finding, which indicates that this type of installation errors should be reduced as small as possible.

\section{References}

[1] I. Kim, Y. H. Kim, I. A. Matisov et al., "Dynamic scheme north finder using a fiber optic gyroscope," in Proceedings of the Fiber Optic Sensors V, pp. 228-236, November 1996.

[2] S. J. Kim, S. S. Lee, Y. S. Kwon, J. C. Bae, and S. J. Lee, "Dynamic north-finding scheme based on a fiber optic gyroscope," in Proceedings of the Navigation and Control Technologies for Unmanned Systems II, pp. 126-136, Orlando, Fla, USA, April 1997.

[3] G. Grenon, P. E. An, S. M. Smith, and A. J. Healey, "Enhancement of the inertial navigation system for the Morpheus autonomous underwater vehicles," IEEE Journal of Oceanic Engineering, vol. 26, no. 4, pp. 548-560, 2001.

[4] O. Celikel, "Application of the vector modulation method to the north finder capability gyroscope as a directional sensor," Measurement Science and Technology, vol. 22, no. 3, Article ID 035203, 2011.

[5] Z. Zhang, J. Sun, and K. Wu, "Error analysis and test study of fiber optic gyroscope north-finder," in Proceedings of the Advanced Sensor Systems and Applications II, pp. 611-618, Beijing, China, November 2004.

[6] T. Tanaka, Y. Igarashi, M. Nara, and T. Yoshino, "Automatic north sensor using a fiber-optic gyroscope," Applied Optics, vol. 33, no. 1, pp. 120-123, 1994.

[7] M. O. Sonnaillon, R. Urteaga, and F. J. Bonetto, "Highfrequency digital lock-in amplifier using random sampling," IEEE Transactions on Instrumentation and Measurement, vol. 57, no. 3, pp. 616-621, 2008.

[8] B. Prasad and R. Lal, "A capacitive immunosensor measurement system with a lock-in amplifier and potentiostatic control by software," Measurement Science and Technology, vol. 10, no. 11, pp. 1097-1104, 1999.

[9] D. S. Ly-Gagnon, S. Tsukamoto, K. Katoh, and K. Kikuchi, "Coherent detection of optical quadrature phase-shift keying signals with carrier phase estimation," Journal of Lightwave Technology, vol. 24, no. 1, pp. 12-20, 2006.

[10] J. H. Choi, J. I. Moon, and S. O. Park, "Measurement of the modulated scattering microwave fields using dual-phase lockin amplifier," IEEE Antennas and Wireless Propagation Letters, vol. 3, no. 1, pp. 340-343, 2004.

[11] W. W. Chow, J. B. Hambenne, T. J. Hutchings, V. E. Sanders, M. Sargent, and M. O. Scully, "Multioscillator laser gyros," IEEE Journal of Quantum Electronics, vol. 16, no. 9, pp. 918-935, 1980.

[12] K. Takiguchi and K. Hotate, "Removal of lock-in phenomenon in optical passive ring-resonator gyros by using optical Kerr effect in fiber resonator," IEEE Photonics Technology Letters, vol. 4, no. 7, pp. 810-812, 1992.

[13] H. J. Bueschelberger, E. Handrich, H. Malthan et al., "Laser gyros in system application with rate-bias technique," in Proceedings of the Sympoisum on Gyro Technology, Stuttgart, Germany, 1987.

[14] D. H. Titterton and J. L. Weston, Strapdown Inertial Navigation Technology, Peter Peregrinis; on Behalf the Institution of Electrical Engineers, London, UK, 1997. 
[15] P. G. Savage, Strapdown Analytics, Strapdown Associates, Maple Plain, Minn, USA, 2000.

[16] K. M. Hays, R. G. Schmidt, W. A. Wilson, J. D. Campbell, D. W. Heckman, and M. P. Gokhale, "A submarine navigator for the 21st century," in Proceedings of the IEEE Position Location and Navigation Symposium, pp. 179-188, April 2002.

[17] D. H. Lee and J. W. Ahn, "Dual speed control scheme of servo drive system for a nonlinear friction compensation," IEEE Transactions on Power Electronics, vol. 23, no. 2, pp. 959-965, 2008.

[18] A. Takano, "Quick-response torque-controlled induction motor drives using phase-locked loop speed control with disturbance compensation," IEEE Transactions on Industrial Electronics, vol. 43, no. 6, pp. 640-646, 1996.

[19] L. Moura and A. Borjak, "A modular phase-frequency detector design with $\pm 2 N \pi$ linear range of operation," Circuits, Systems, and Signal Processing, vol. 23, no. 4, pp. 273-283, 2004.

[20] M. T. Hill and A. Cantoni, "A digital implementation of a frequency steered phase locked loop," IEEE Transactions on Circuits and Systems I, vol. 47, no. 6, pp. 818-824, 2000.

[21] L. C. Ng and D. J. Pines, "Characterization of ring laser gyro performance using the allan variance method," Journal of Guidance, Control, and Dynamics, vol. 20, no. 1, pp. 211-214, 1997.

[22] A. V. Oppenheim, A. S. Willsky, and S. H. Nawab, Signals and Systems, Prentice-Hall, Upper Saddle River, NJ, USA, 2nd edition, 1997.

[23] A. Mehrotra, "Noise analysis of phase-locked loops," IEEE Transactions on Circuits and Systems I, vol. 49, no. 9, pp. 13091316, 2002.

[24] S. Qin, Z. Huang, and X. Wang, "Optical angular encoder installation error measurement and calibration by ring laser gyroscope," IEEE Transactions on Instrumentation and Measurement, vol. 59, no. 3, pp. 506-511, 2010. 


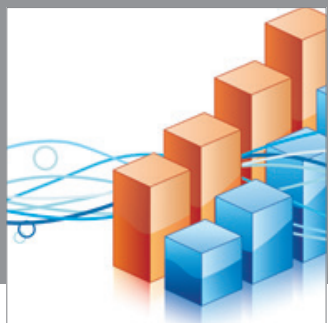

Advances in

Operations Research

mansans

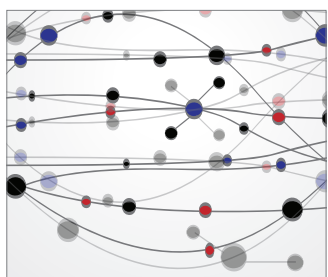

The Scientific World Journal
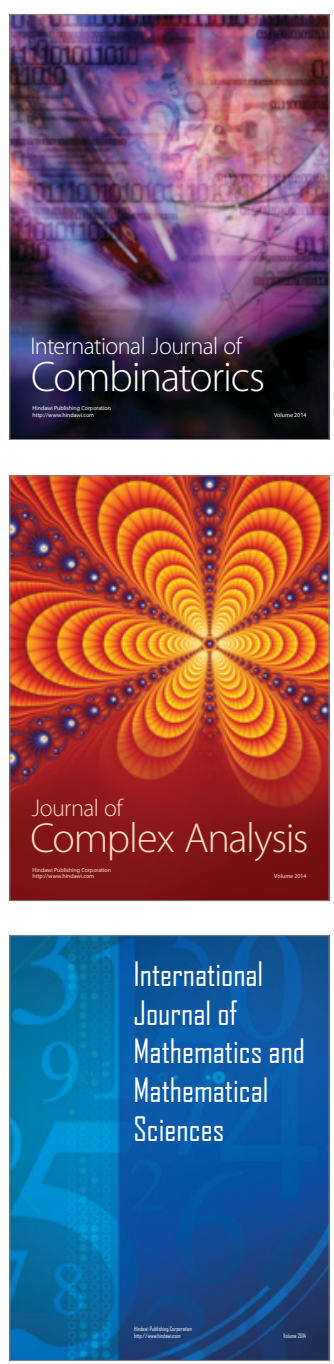
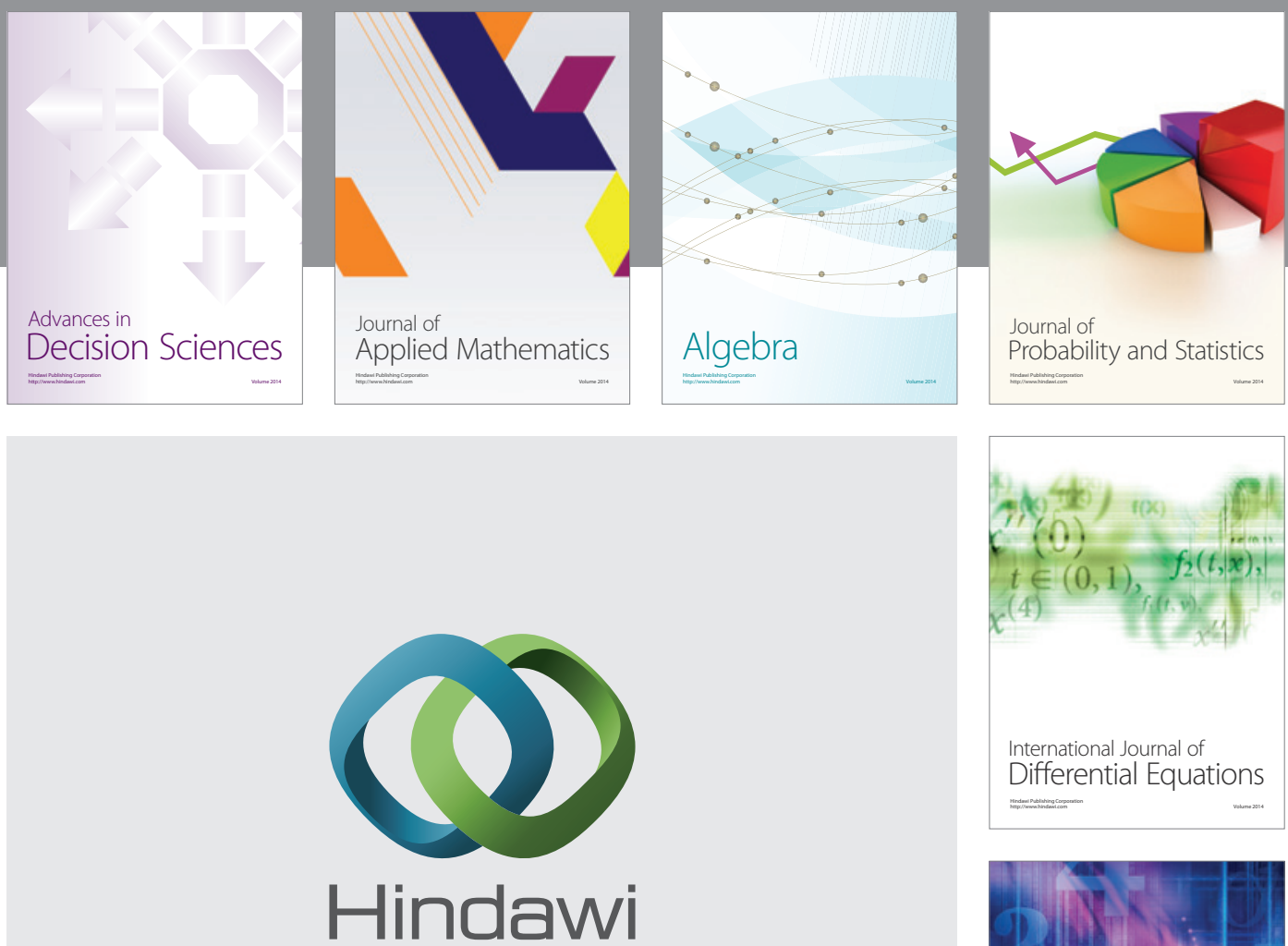

Submit your manuscripts at http://www.hindawi.com
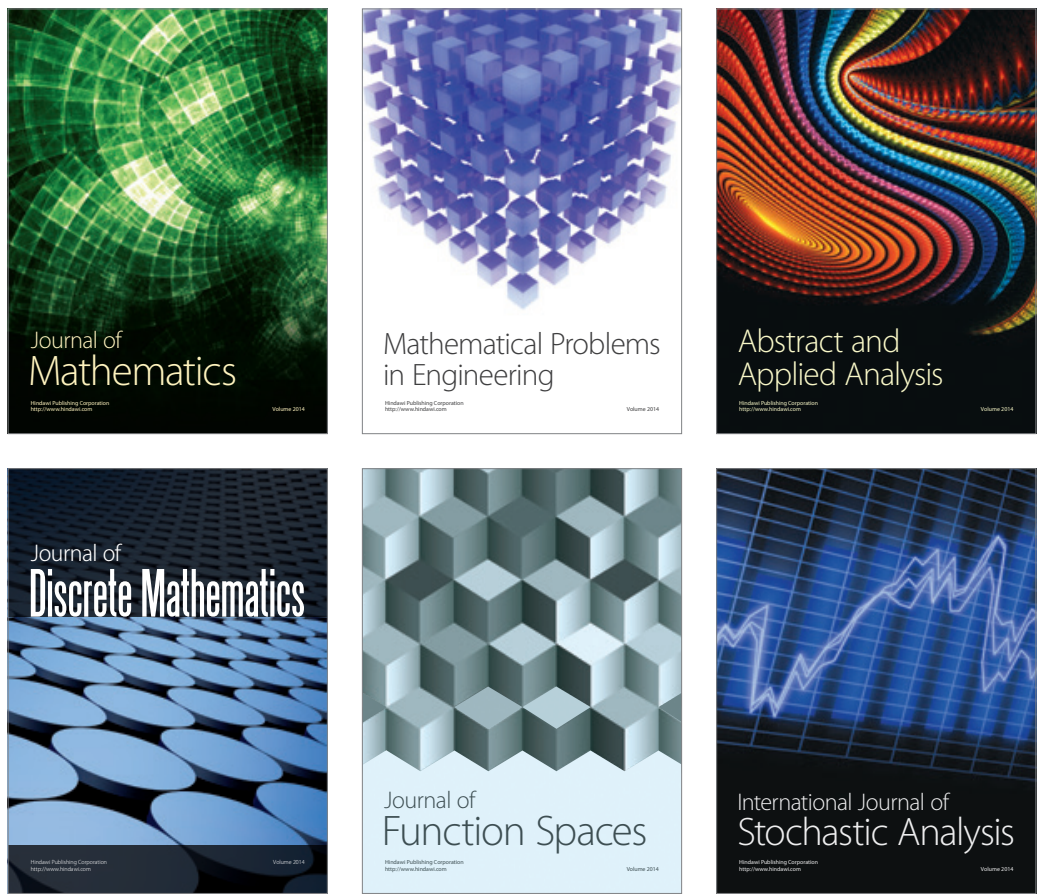

Journal of

Function Spaces

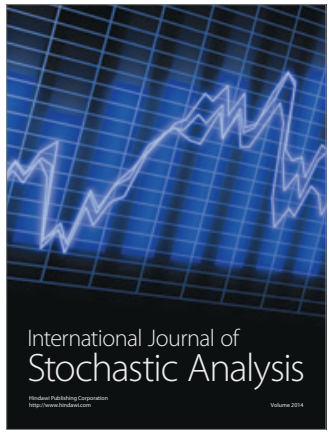

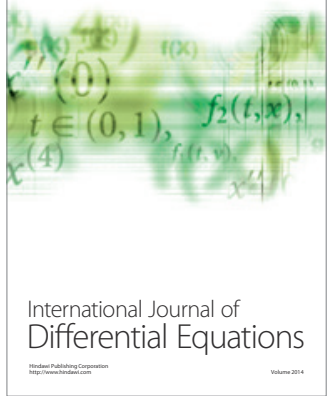
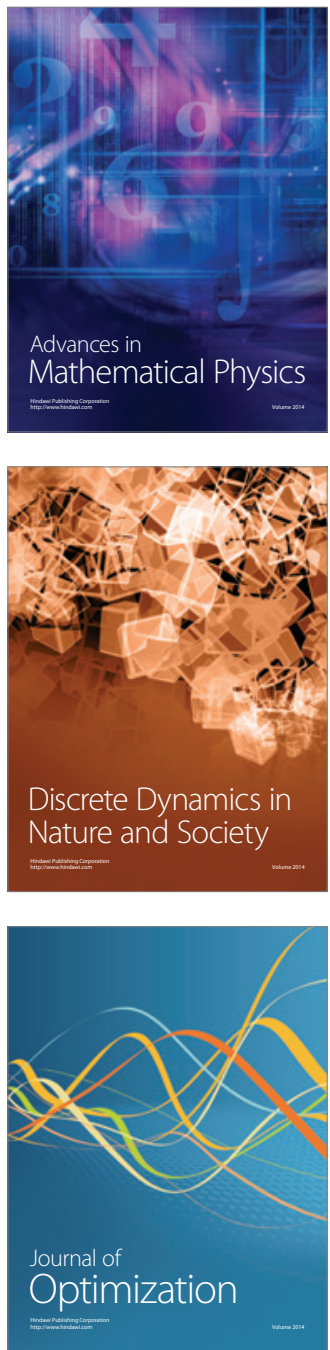